\title{
Filigrane
}

Écoutes psychothérapiques

\section{Brève étude sur la perception, l'inconscient et le temps}

\section{Dominique Scarfone}

Volume 22, numéro 1, printemps 2013

Psychanalyse et temporalités

URI : https://id.erudit.org/iderudit/1017344ar

DOI : https://doi.org/10.7202/1017344ar

Aller au sommaire du numéro

Éditeur(s)

Revue Santé mentale au Québec

ISSN

1192-1412 (imprimé)

1911-4656 (numérique)

Découvrir la revue

Citer cet article

Scarfone, D. (2013). Brève étude sur la perception, l'inconscient et le temps. Filigrane, 22(1), 27-37. https://doi.org/10.7202/1017344ar

\section{Résumé de l'article}

L'auteur propose ici une " étude ", c'est-à-dire une exploration de la concordance et de la différence qui peut exister entre, d'une part, la conception énactive de la perception en philosophie de l'esprit (Alva Noë) et, d'autre part, la conception métapsychologique de la perception de l'autre humain, qui va du Projet de Freud à la théorie de la séduction généralisée de Laplanche. Par cette mise en parallèle de deux théories de la perception, l'auteur croit pouvoir étayer une proposition concernant la temporalité particulière de l'inconscient : un temps " actuel ». C'est une proposition dont il a commencé l'élaboration il y a quelques années et qu'il poursuit ici sous un nouvel angle. 


\section{Brève étude sur la perception, I'inconscient et le temps}

\section{Dominique Scarfone}

L'auteur propose ici une "étude", c'est-à-dire une exploration de la concordance et de la différence qui peut exister entre, d'une part, la conception énactive de la perception en philosophie de l'esprit (Alva Noë) et, d'autre part, la conception métapsychologique de la perception de I'autre humain, qui va du Projet de Freud à la théorie de la séduction généralisée de Laplanche. Par cette mise en parallèle de deux théories de la perception, l'auteur croit pouvoir étayer une proposition concernant la temporalité particulière de l'inconscient: un temps "actuel». C'est une proposition dont il a commencé l'élaboration il y a quelques années et qu'il poursuit ici sous un nouvel angle.

n certain accord semble se faire en psychanalyse, ouvertement ou tacitement, au sujet de l'atemporalité de l'inconscient tel que Freud (1915) l'a posée dans son texte sur «L'inconscient». Attribuer un temps à l'inconscient pourrait donc sembler prendre le contre-pied de Freud. Pour ma part, j'en suis venu à penser que, à ce sujet comme à bien d'autres, les choses ne sont pas aussi simples qu'elles en ont l'air et que l'on peut rester dans la ligne de pensée de Freud tout en parlant d'une sorte de temporalité inconsciente. Encore faut-il expliciter ce que serait cette temporalité. Mais si, comme l'affirme et l'argumente le philosophe de l'esprit Alva Noë dans son plus récent livre (2012), percevoir quelque chose signifie en fait avoir le sentiment que la chose perçue nous est accessible, c'est-à-dire que nous pouvons en avoir un certain usage (théorie énactive de la perception), alors je voudrais tenter, sur cette base, de faire quelques pas dans la réflexion sur le «temps actuel» que, pour ma part, j'identifie comme le temps de la "chose inconsciente». Réflexion qui sous des allures toutes théoriques a selon moi de fortes incidences sur la pratique psychanalytique, comme je tenterai de l'indiquer. 


\section{Perception}

Pour commencer, notons que, dans l'optique de Noë, « avoir un certain usage» de la chose perçue, cela signifie la comprendre. Déjà Wittgenstein (1953, proposition 43) avait mis un signe d'égalité entre sens et usage. En raison de quoi, la perception, comme Noë s'en explique parfaitement, n'a rien à voir avec un quelconque modèle photographique qui produirait des représentations internes du monde perçu. Pour lui, mais tout aussi bien pour Freud $(1895,1925)$, la perception est une activité, c'est quelque chose que nous faisons, c'est une disposition de notre psyché-corps en relation avec le perçu. En suivant à la trace la pensée de Freud à ce sujet, Marie Leclaire et moi avons pu établir qu'il n'y a pas de perception authentique, avec son sens de réalité, sans l'activation des images motrices (Leclaire et Scarfone, 2000). Cela signifie que le sujet qui perçoit agit sur le monde autour de lui et que son corps (ses sens, mais aussi ses muscles) s'active, ne serait-ce que virtuellement, pour faire usage de ce monde. Le travail afin d'obtenir cette compréhension, cette accessibilité, c'est l'intentionnalité de la perception. Puisque la perception est un acte, elle s'inscrit d'emblée dans un "arc intentionnel» (Merleau-Ponty, 1945) qui saisit la chose perçue dans son ensemble, tout d'un coup, y compris, ajoute Noë, dans les aspects des objets qui sont cachés à nos sens. Ainsi, lorsque nous regardons une pomme nous ne pouvons en voir qu'une face, et cependant nous faisons l'expérience visuelle, nous dit Noë, non d'une moitié de pomme, mais d'une pomme entière. Les choses se présentent donc à nous entières dans la perception, y compris dans ce qu'elles comportent d'absence.

Ce schéma relativement simple concernant la perception se complique cependant lorsque la perception qui nous occupe n'est pas la perception de l'objet quelconque - qui intéresse les philosophes de l'esprit -, mais celle de l'autre humain et, puisque nous parlons de psychanalyse, pas n'importe quel autre humain. Il s'agit de l'autre secourable, celui que Freud, dans son Projet (1895) a appelé le Nebenmensch, soit, à la lettre, l'humain proche, le semblable, le voisin, le prochain. Pour l'infans, ce sera la mère ou toute personne en tenant lieu. Ici, les choses se compliquent un peu, parce que plus d'un siècle avant l'identification des "neurones miroirs", il était bien connu de Freud que la perception du semblable avait d'emblée une valeur d'imitation et une valeur d'empathie. Autrement dit, «pendant que l'on perçoit [...], on imite le mouvement lui-même [...] Ou bien la perception réveille l'image mnésique d'une sensation de douleur propre [au sujet] ; on ressent alors le déplaisir correspondant...» (Freud, 1895, 641). Cela signifie que l'autre humain 
ainsi perçu est «compris» (p. 639). Cette compréhension imitative et empathique va bien au-delà de la compréhension de l'objet inerte décrite plus haut et on pourrait la croire plus riche, plus complète. Mais il y a un hic. C'est que le «complexe de perception » concernant cet autre familier, alors même qu'il comporte apparemment cette expérience d'une saisie totale, présente néanmoins lui aussi une part "absente », une part qui résiste à la compréhension, c'est-à-dire à l'imitation et à l'empathie, compagnes de la perception. D'une part, nous sommes confortés par ce parallélisme entre la perception en général d'un objet quelconque et celle d'un de nos semblables; mais d'autre part, la différence va nous apprendre plus de choses importantes pour la psychanalyse que la perception en général.

Freud dit de cette partie qui résiste qu'elle "se [soustrait] au jugement» (p. 642) et il l'appelle « la chose» (das Ding). La « chose» se caractérise par sa «structure constante ", alors que la part compréhensible, imitable, est quant à elle, variable. Ce sont d'ailleurs ses variations qui permettent au sujet de s'en faire un compte rendu compréhensible, par la possibilité de s'en référer à l'expérience imitative de son propre corps. Lacan a relevé cette notion de chose et l'a commentée longuement dans son séminaire sur l'éthique (Lacan, 1959-1960). Il n'y a pas lieu ici de reprendre ce qu'il en dit, sinon pour souligner cette remarque: "Das Ding [la chose] est originellement ce que nous appellerons le hors-signifié» (p. 67), ce qui concorde parfaitement avec ce que Freud exprimait en disant qu'elle correspond à ce qui se soustrait au jugement, n'est pas compréhensible; donc, que celui qui la perçoit n'en a pas pour autant l'usage. Pour Lacan c'est l'objet perdu, «[...] Autre absolu du sujet qu'il s'agit de retrouver» (p. 65) en tant qu'il est inoubliable; et cependant cet objet est impossible à retrouver, sauf «tout au plus en tant que regret» (ibid.).

Nous verrons plus loin, avec un des premiers élèves de Lacan, à quoi peut se ramener plus précisément cette chose en son altérité, cette part échappant à la capacité de compréhension et de reconnaissance. Mais pour l'instant, éloignons-nous de cet aspect et soulignons la congruence entre la perception de la partie compréhensible de l'autre humain et tout ce qui est étudié dans le cadre de la théorie de l'attachement. Il ne fait aucun doute, en effet, que l'accordage dont parlent les développementalistes, par exemple, correspond à la compréhension mutuelle entre la mère (l'adulte) et l'enfant. D'ailleurs il est tout à fait acceptable, même pour la théorie psychanalytique, de postuler l'existence de montages instinctuels, innés, résultant d'une longue évolution au sein de l'espèce et favorisant cet ajustement, cette communication relativement claire entre la mère et l'enfant. Il n'est pas difficile d'imaginer, en 
termes de sélection naturelle, les avantages de cet accord pour la survie de l'espèce.

Mais, disions-nous, il y a un hic. Or ce hic nous le retrouvons fiché au beau milieu de l'attachement comme précisément ce qui constitue la ligne de démarcation entre le champ de l'attachement - ou plus généralement, de l'éthologie humaine - et le champ psychanalytique. Le hic c'est que, alors même que le petit humain est exposé à un monde adulte dont une partie lui est assez facilement compréhensible, au sein de laquelle il s'adaptera tant bien que mal au fur et à mesure que les boucles de rétroaction avec l'autre familier confirmeront sa compréhension, devenant ainsi de plus en plus efficaces, il y a par ailleurs cette autre partie qui ne peut qu'échapper à sa compréhension, à son jugement. On reconnaît là ce qu'on disait plus haut de «la chose ", mais au lieu de la poser dans l'abstrait de la perception en général, demandons-nous quelle est donc la part de l'autre adulte que l'infans ne peut absolument pas comprendre. Cette part, c'est le sexuel refoulé de l'adulte - refoulé, mais non inactif -, un sexuel d'autant plus susceptible d'échapper au jugement de l'infans que chez l'adulte lui-même, il échappe à sa maîtrise consciente. Selon Laplanche (1987), l'adulte même le mieux ajusté aux besoins de l'enfant est néanmoins émetteur malgré lui de messages énigmatiques, c'est-à-dire que ses messages les mieux formés sont néanmoins « compromis » par le sexuel refoulé dont il est porteur. Les fonctions d'attachement les plus normales sont alors en même temps comme les ondes porteuses de cette part "compromise», énigmatique pour l'adulte lui-même qui en est l'émetteur inconscient.

Voilà donc, si nous sortons de la théorie générale de la connaissance et que nous nous en tenons à l'expérience analytique, ce que serait la «chose » signifiée par Freud dans le Projet; voilà ce que l'enfant capte obscurément mais dont il n'a pas l'usage. Le petit humain vient en effet au monde dans un état de prématurité que les soins maternels (parentaux) se chargent de pallier; mais il est une prématurité, sexuelle, qui ne sera surmontée biologiquement que beaucoup plus tard chez cet enfant avec la maturité atteinte à la puberté. Or, quand elle viendra, cette maturité sexuelle biologiquement programmée, le sexuel infantile en aura déjà occupé la place pour ne plus en être délogé. Cette divergence fondamentale entre sexuel infantile et sexualité biologique, c'est ce qui met l'enfant humain dans une position tout à fait originale par rapport aux petits d'autres espèces: comme le souligne Laplanche, l'acquis arrive ici avant l'inné. Encore faut-il comprendre comment se forme cet acquis qu'est le sexuel infantile. 


\section{Inconscient}

Revenons brièvement à Alva Noë et distinguons avec lui entre perception d'un objet inerte et perception d'un événement. Nous nous accorderons alors sur ceci que ce que l'enfant reçoit de l'adulte et dont il n'a pas l'usage n'est pas un objet inerte. Même dans le cas où un tel objet lui serait présenté, donné à voir, la structure nécessairement relationnelle dans laquelle sont insérés l'adulte et l'enfant fait en sorte qu'il y a là non seulement un objet matériel à voir, à entendre, à toucher, mais un événement qui fait signe. Il y a là, en effet, une intentionnalité, même si elle n'est pas consciente; l'enfant la capte bien dans sa nature d'événement et y répond, pour une part, avec une bonne compréhension. Dans une situation d'allaitement, par exemple, l'enfant prend le mamelon dans sa bouche et commence à téter. Il utilise correctement l'objet concret qui lui est présenté. Ce dont il ne sait que faire, cependant, c'est cette autre dimension de l'événement: il s'agit de ce que la mère vit et produit inconsciemment, qui est d'ordre sexuel et qui va au-delà de ce qui entre dans les boucles de rétroaction communicationnelles auxquelles l'enfant contribue ordinairement. À cette dimension sexuelle de la communication maternelle, l'enfant n'a pas de réponse adéquate. Quelque chose se communique qui reste opaque pour l'enfant, quelque chose dont il n'a pas l'usage: message énigmatique, parce que «compromis» de sexuel inconscient, prenant origine dans le refoulé maternel. On pourrait là-dessus objecter que si on présente au nourrisson un clavier d'ordinateur, il n'en saura pas quoi faire non plus. Mais cela n'est qu'une affaire de temps et on peut d'ailleurs ajuster les difficultés d'un ordinateur aux capacités progressives de l'enfant. Mais pour le sexuel inconscient, il n'y a pas d'ajustement possible; d'autant plus que, comme déjà souligné, l'énigme est énigme pour l'adulte également.

Je signale tout de suite que l'exemple ci-dessus est, comme tout exemple, un peu boiteux, parce qu'en fait toutes les communications entre les adultes et l'infans sont ainsi chargées d'une dimension sexuelle inévitable. J'ai pris l'exemple de l'allaitement pour bien faire ressortir la dimension sexuelle, mais dans un sens ce serait trompeur de penser les choses seulement à partir de situations où la dite dimension sexuelle serait aussi patente. Il faut plutôt considérer que toute la vie de l'enfant est toujours déjà inscrite dans l'ordre du sexuel: de sa conception à sa naissance, aux manifestations tendres et passionnées dont il est l'objet au cours des soins qu'on lui prodigue et qui impliquent des désirs, des fantasmes, un excitation de la surface érogène du corps (pas seulement celle de l'enfant d'ailleurs), etc. Autrement dit, le sexuel 
inconscient n'est pas quelque chose qui se surajouterait à la dimension d'attachement. Celle-ci est toujours déjà «compromise» par le sexuel; pour le petit humain il n'existe pas une situation d'attachement "pur». Le sexuel est, pour l'enfant, la part incompréhensible du «complexe de perception » que constitue sa mère, son père ou tout adulte lui prodiguant des soins.

Quelque chose s'inscrit là, même si l'enfant n'en a pas l'usage. Cette inscription, Laplanche (1987) l'a pensée comme «implantation » du sexuel, dans le cadre de la théorie de la séduction généralisée. Implantation qu'on aurait tort de penser comme une sorte de transcendance immatérielle ou purement «structurale». L'implantation résulte du décalage effectif entre les adultes et l'enfant dans la «situation anthropologique fondamentale» où se retrouve l'enfant humain dès sa naissance. Cette situation contient l'attachement, mais ne s'y limite pas, puisqu'elle comporte le gradient, la différence décisive entre adulte et infans, soit la présence chez l'adulte d'un sexuel refoulé, sexuel absent chez l'infans qui subit cependant obscurément l'impact du sexuel de l'autre.

Récapitulons: il y a perception d'un quelque chose qui a un effet mais dont l'enfant n'a pas l'usage, ni donc la compréhension. Que se passera-t-il à partir de là?

Il se passera que le petit humain est doué d'une tendance irrépressible à comprendre, à construire activement le sens, c'est-à-dire, comme on l'a vu, à acquérir l'usage de ce qu'il perçoit. Il se mettra donc en quête de la signification de ce qui l'affecte, qui lui fait signe mais dont le sens lui demeure étranger. Ce dont il n'a pas l'usage adéquat - le sexuel - s'implante donc en tant qu'épine irritative, comme une chose qui ne se plie pas au sens, ou alors seulement en partie, et qui incite le petit humain à un effort constant de maîtrise, de traduction. Mais la fonction herméneutique, traductive, ainsi mise en branle se heurte à répétition à cet obstacle de l'opacité de la part énigmatique du message. L'enfant se débrouille comme il peut, se fiant notamment à l'expérience de son propre corps, avec les moyens du bord. Ainsi, il se construira ses propres théories sexuelles infantiles. La traduction ne réussira que partiellement, laissant des scories importantes. Le sens que l'enfant aura néanmoins construit, aussi partiel soit-il, constituera un noyau du moi, noyau appelé à se combiner à d'autres noyaux semblables pour donner un moi «cohérent». Les restes non-traduits, eux, constitueront le refoulé originaire. Freud avait lui-même postulé que le refoulement n'est rien d'autre qu'un défaut ou un refusement de traduction (Lettre à Fliess du 6/12/1896). Ce reste-là, donc, ne s'intégrera pas au moi mais constituera le noyau de l'inconscient ${ }^{1}$, cet inconscient dont Freud dira qu'il est a-temporel (zeitlos). 


\section{Temps}

Nous voici donc parvenus à la question que nous posions dans notre titre mais dont nous n'avons pas encore traité: la question du temps.

Revenons une fois de plus à Alva Noë. Cet auteur très rigoureux, travaillant du point de vue de la philosophie de l'esprit, dit à un moment donné cette chose étonnante: que les choses perçues - il veut alors dire les objets inertes, une chaise, une pomme - sont d'une certaine façon a-temporelles (timeless). Il explique que c'est dans le sens où elles sont posées là, devant nous et qu'elles « existent d'un seul trait, entières et intégrées » (they exist, all at once, whole and integrated) (Noë, 2012, 74). Bien entendu, Noë ne contesterait pas que le temps finirait par affecter cette existence. Il semble donc ne considérer qu'un horizon de temps assez bref, le temps de la perception, pour dire qu'elles sont a-temporelles. Il y aurait peut-être lieu ici d'introduire la distinction bergsonienne entre temps et durée (Bergson, 1889), mais nous n'entrerons pas dans cette discussion. Acceptons pour l'instant cette idée d'a-temporalité de la chose perçue, lorsqu'il s'agit d'un objet inerte, puisque, comme je l'ai déjà souligné, en psychanalyse ce n'est pas ce type de perception qui nous importe. Nous l'acceptons, entre autres raisons, parce que cette a-temporalité sert à Noë pour distinguer la perception d'une chose de la perception d'un événement. Ce qui les distingue, c'est qu'un événement est nécessairement inscrit dans le temps. En fait, par contraste avec les choses inertes, la dimension temporelle est consubstantielle à l'événement, faute de quoi il n'en resterait pas grand chose. La pomme est là, devant moi, immobile et j'ai tout le loisir, dans un horizon de temps donné, mais néanmoins assez durable, d'en faire le tour si je veux, de la contempler à mon aise: le temps peut pour moi «suspendre son vol» (alors même qu'un observateur muni d'un chronomètre pourrait mesurer la durée de mon observation). Mais si quelqu'un me parle ou s'adresse à moi d'une quelconque façon, cela fait événement, cela s'inscrit par conséquent dans une séquence, dans une alternance déictique où mon interlocuteur et moi échangeons successivement nos rôles ou nos places dans l'interlocution: tantôt il parle et j'écoute (il est le « je», je suis le «tu»); tantôt je parle et il m'écoute. Cette succession est nécessaire si l'on veut se comprendre, elle doit s'écouler le long d'une ligne de temps (diachronie), parce que si nous parlions en même temps (synchronie) nous ne nous comprendrions pas. Il est donc possible de conclure que lorsque mon interlocuteur et moi successivement parlons, écoutons et comprenons, nous sommes nécessairement dans une dimension temporelle (Lyotard, 1990). 
Si nous revenons maintenant à l'infans exposé aux messages énigmatiques de l'autre humain, nous en déduirons que, pour la part du message qu'il est capable de traduire, de comprendre (l'aspect attachement, par exemple), le temps entrera en ligne de compte. Déjà, les vocalises que mère et bébé s'échangent établissent cette permutation déictique dont nous parlions à l'instant. Mais qu'en est-il de la dimension du message non comprise, non assimilable au moi? L'impact de cette partie est certes enregistré, mais le fait que l'enfant ne puisse en faire usage, qu'elle soit donc mise en réserve en tant que noyau inconscient, en tant que "à traduire», cela lui donne, comme nous l'avons vu, le statut de «chose». En tant que chose, il nous faut la considérer, tout comme Noë le faisait des choses inertes dans la perception, comme atemporelle, dans le sens où elle ne s'est pas inscrite dans une alternance déictique: il n'y a pas à son sujet de séquence, d'interlocution possible. Nous retrouvons ainsi la «chose» du Projet, chose pour laquelle, même adultes, les mots nous font défaut. Souvenons-nous que, selon le mot de Lacan, la chose, c'est «le hors-signifié »; il n’y a pas de mots pour la dire et nous voilà donc littéralement dans l'in-fantia, si l'on pense que «in-fans» désigne justement celui qui ne parle pas parce que ne disposant pas encore des mots nécessaires.

Fort bien! Mais nous avons vu que, puisque en psychanalyse, nous nous intéressons non pas à la perception d'objets inertes mais à la perception d'événements, de communication inter-humaine, alors dans la logique de Noë il faudrait aussi que l'événement perçu soit d'emblée d'ordre temporel. Comment résoudre cette apparente contradiction? Comment concilier les deux aspects de ce perçu qui d'une part est «chose» mais n'est pas inerte; qui d'autre part est événement, mais sans séquence?

Je proposerai que la contradiction se résout de la façon suivante: nous avons admis avec Noë que la dimension temporelle est essentielle à tout événement, contrairement à ce qui se passe pour les choses inertes. Cependant, nous avons vu que l'inscription dans le temps chronologique (dans la diachronie) suppose deux sujets en mesure d'échanger leur place et leur rôle dans l'interlocution. Or, nous avons également vu que pour l'enfant, ce « dialogue» n'est possible que pour la dimension «attachement» de la relation, mais nullement pour la dimension sexuelle. En effet, cette dimension lui demeure opaque, il n'en a pas l'usage, il ne peut l'intégrer. Toutefois, l'aspect «événement» vaut tout autant pour cette partie non assimilable et incompréhensible que pour la partie «bien ajustée» de l'attachement. Voici donc que l'implantation du sexuel inconscient est à la fois événementielle, donc temporelle, et hors diachronie! Hors diachronie, dirons-nous, mais pas 
exactement a-temporelle, puisque cette partie s'inscrit néanmoins dans la durée et qu'elle agit en permanence aux frontières du moi cohérent, poussant à l'effort de traduction. La séquence temporelle fait défaut, mais la dimension intentionnelle (au sens de la phénoménologie) demeure et avec elle, nécessairement, une forme de temporalité.

Cette chose inconsciente, par conséquent, n'est pas absolument a-temporelle comme le serait une chose inerte (au sens de Noë). Nous lui donnerons par conséquent une temporalité bien à elle en la qualifiant d' "actuelle » (Leclaire et Scarfone, 2000; Scarfone, 2006). Actuelle dans plus d'un sens: «actuelle» parce que toujours là et ne passant pas. Actuelle parce que sa traduction ne sera jamais complète, toujours à reprendre; objet toujours à retrouver, mais qui ne le sera jamais vraiment. «Actuelle» parce que son temps ne passe pas (Pontalis, 1997), que son heure est toujours «maintenant» (Lyotard, 1990). «Actuelle» enfin parce que en acte, c'est-à-dire parce que non encore pleinement psychique, à la manière de ces noyaux de «névrose actuelle» que Freud posait au cœur des psychonévroses de transfert même les plus élaborées (Freud, 1917; de M’Uzan, 1970).

\section{Conséquences pour la conduite de la cure}

Il s'ensuit que l'inconscient dans son sens le plus strict n'est pas fait de représentations bien formées, mais de choses "actuelles»; des "choses» poussant vers la représentation, mais se heurtant toujours à la limite qu'est l'impossibilité de traduire intégralement cette «chose». Difficulté d'autant plus grande que désormais le moi cohérent opposera lui-même une résistance à toute traduction qui, dans l'après-coup, pourrait exiger une remaniement de la cohérence péniblement acquise. Cohérent, en effet, ne veut pas dire gardien de la vérité, et bien des compromis et des compromissions seront acceptés par le moi afin de maintenir sa cohérence.

Si donc l'inconscient est fait de ces noyaux «chosiques », restes d'événements incomplètement compris, traduits ou assimilés, sur quoi travaille donc l'analyse lorsqu'elle... analyse? Bien entendu, il ne saurait y avoir d'accès direct à cet inconscient. Ce que l'analysant apporte en séance, ce sont ses traductions préalables, ses façons plus ou moins boiteuses de s'arranger avec les énigmes auxquelles il a été confronté depuis l'enfance: ses symptômes, ses compulsions à répéter, etc. C'est cela que l'analyse devra décomposer (c'est le sens exact d'analyser). Une conception traductive de la constitution de l'inconscient ne saurait par conséquent impliquer que l'analyste se ferait, quant à lui, traducteur pour son patient. L'institution du cadre analytique signifie 
la mise en suspens de l'adaptatif (qui correspondrait à la dimension «bien ajustée » de la communication entre l'adulte et l'infans). Il n'est donc pas dit qu'analyste et analysant devraient d'emblée «se comprendre». C'est même tout l'opposé qui est vrai. L'analyste n'est pas là pour tout comprendre - cela, c'est le fantasme du patient: l'analyste comme sujet supposé savoir, selon l'expression de Lacan. L'analyste doit écouter et savoir entendre, entendre notamment ce qui s'emboîte mal, ce qui grince dans les histoires, les théories, les fantasmes et autres traductions que le patient apporte et qui l'ont conduit à une impasse. Les points où «ça grince» feront l'objet d'un « faire signe » de l'analyste qui incitera ainsi indirectement l'analysant à poursuivre plus avant sa "dé-traduction", sa déconstruction de ce qu'il avait déjà construit. C'est un travail de déblayage qui creuse progressivement un nid pour le transfert, creux de signification où se reconstituera - toutes proportions gardées - la situation anthropologique fondamentale: l'analysant sera confronté à l'énigme de l'analyste tout comme l'infans l'était à celle de l'adulte, réactivant ainsi les traces mnésiques, les «choses» inconscientes en manque de mots, de représentation (Laplanche, 1991). Une analyse bien menée dans le sens de cette déconstruction conduira par conséquent vers des lieux inattendus, non cartographiés, que l'analyste, pas plus que son patient, ne saurait ni ne devrait prévoir (Scarfone, 2012).

Les «choses» inconscientes qui se présenteront alors (avant de pouvoir être représentées), se présenteront «en acte», puisque «actuelles» comme nous disions. Le transfert sera précisément leur mise en acte. Si tout se passe bien, une part de ces choses inconscientes pourra être traduite de manière plus large, plus souple, plus en phase avec la vie présente de l'analysant que ne l'avaient fait les traductions antérieures. Ces traductions nouvelles et plus englobantes guideront les choix que le patient pourra désormais faire quant à la question de «comment vivre». Cela implique une position éthique rigoureuse de la part de l'analyste, notamment, que celui-ci se garde de «savoir» ce que serait la bonne traduction, donc les bons choix, et qu'il laisse au traducteur qu'est l'analysant le soin de se faire une nouvelle version de son histoire, de sa position dans le réseau de relations avec les autres. Cela ne signifie pas qu'il y aurait une traduction finale, le sexuel inconscient étant cette chose par définition non complètement maîtrisable, toujours actuelle, et qui nous trouve toujours non préparés, toujours en manque de représentations, toujours in-fans.

Dominique Scarfone dominique.scarfone@umontreal.ca 


\section{Note}

1. Pour ne pas trop compliquer le tableau, je fais abstraction de la différence qu'il faudrait faire entre un inconscient résultant du refoulement originaire et ce qui résulte d'un refoulement secondaire.

\section{Références}

BERGSON, H., 1889, Essai sur les données immédiates de la conscience, Paris, PUF.

FREUD, S., 1895, Projet d'une psychologie, in Lettres à Wilhelm Fliess, Paris, PUF, 2006.

FREUD, S., 1915, L'inconscient, Euvres complètes, vol. XIII, Paris, PUF.

FREUD, S., 1917, Conférences d'introduction à la psychanalyse, Euvres complètes, vol. XIV, Paris, PUF.

FREUD, S., 1925, La négation, in Résultats, idées, problèmes, II, Paris PUF, 1984.

FREUD, S., 1925, Le Bloc-notes magique, in Résultats, idées, problèmes, II, Paris PUF, 1984.

LACAN, J., 1959-1960, Le Séminaire, livre VII - L’Éthique de la psychanalyse, Paris, Seuil, 1986.

LAPLANCHE, J., 1987, Nouveaux fondements pour la psychanalyse, Paris, PUF.

LAPLANCHE, J., 1991, Du transfert: sa provocation par l'analyste, in Le primat de l'autre en psychanalyse (Textes 1967-1991), Paris, Flammarion, 1997, 417-437.

LECLAIRE, M., SCARFONE, D., 2000, Vers une conception unitaire de l'épreuve de réalité, Revue française de psychanalyse, LXIV, 3, 885-912.

LYOTARD, J.-F., 1990, Les voix d'une voix, in Histoires de cas, Nouvelle revue de psychanalyse, $\mathrm{n}^{\circ} 42$; 1991, Voix : Freud, in Lectures d'enfance, Paris, Galilée, 129-153.

MERLEAU-PONTY, M., 1945, Phénoménologie de la perception, Paris, Gallimard.

M'UZAN, M. De, 1970, Le même et l'identique, in De l'art à la mort, Paris, Gallimard.

NOË, A., 2012, Varieties of Presence, Cambridge, Harvard University Press.

PONTALIS, J.-B., 1997, Ce temps qui ne passe pas, Paris, Gallimard.

SCARFONE, D., 2000, Sexuel et actuel, in D. Widlöcher, J. Laplanche et coll., Sexualité infantile et attachement, Paris, PUF.

SCARFONE, D., 2006, Un temps sans mémoire, in B. Chouvier et R. Roussillon (sous la direction de), La temporalité psychique, Paris, Dunod.

SCARFONE, D., 2012, Quartiers aux rues sans nom, Paris, Éditions de l'Olivier.

WITTGENSTEIN, L., 1953, Investigations philosophiques, publié en français à la suite du Tractatus logico-philosophicus, Paris, Gallimard, 1961. 